\title{
Resonant plasmonic nanoparticles for multicolor second harmonic imaging
}

Article in Applied Physics Letters · February 2016

DOI: $10.1063 / 1.4942902$

CITATIONS

2

5 authors, including:

\section{Nicolò Accanto}

ICFO Institute of Photonic Sciences

13 PUBLICATIONS 95 CITATIONS

SEE PROFILE

\section{Jan Renger}

Max-Planck-Institut für die Physik des Lichts 48 PUBLICATIONS 1,327 CITATIONS

SEE PROFILE

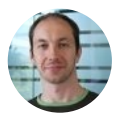

Lukasz Piatkowski

ICFO Institute of Photonic Sciences

32 PUBLICATIONS 360 CITATIONS

SEE PROFILE

Niek F Van Hulst

ICFO Institute of Photonic Sciences

400 PUBLICATIONS 13,112 CITATIONS

SEE PROFILE 


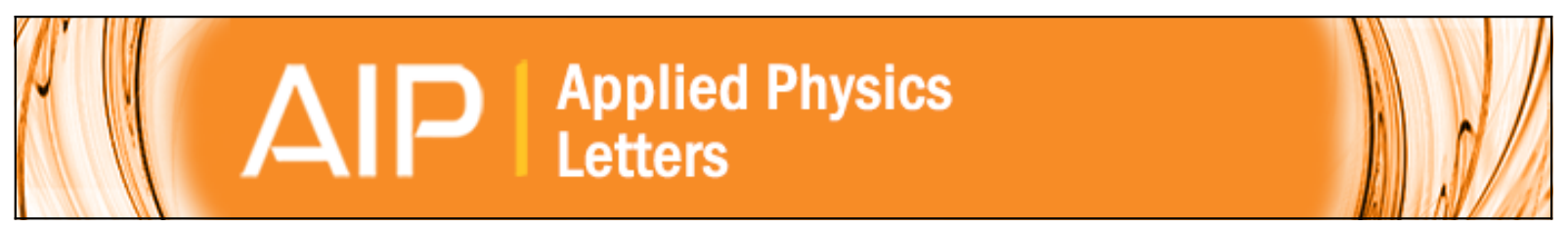

\section{Resonant plasmonic nanoparticles for multicolor second harmonic imaging}

Nicolò Accanto, Lukasz Piatkowski, Ion M. Hancu, Jan Renger, and Niek F. van Hulst

Citation: Applied Physics Letters 108, 083115 (2016); doi: 10.1063/1.4942902

View online: http://dx.doi.org/10.1063/1.4942902

View Table of Contents: http://scitation.aip.org/content/aip/journal/apl/108/8?ver=pdfcov

Published by the AIP Publishing

\section{Articles you may be interested in}

Nanostructure induced changes in lifetime and enhanced second-harmonic response of organic-plasmonic hybrids

Appl. Phys. Lett. 107, 251102 (2015); 10.1063/1.4938007

Enhanced multicolor fluorescence in bioimaging using deep-ultraviolet surface plasmon resonance Appl. Phys. Lett. 104, 223703 (2014); 10.1063/1.4881325

Highly confined, enhanced surface fluorescence imaging with two-dimensional silver nanoparticle sheets Appl. Phys. Lett. 104, 121906 (2014); 10.1063/1.4869560

Third-order nonlinear optical response of CulnS2 quantum dots-Bright probes for near-infrared biodetection Appl. Phys. Lett. 102, 243702 (2013); 10.1063/1.4811786

Enhanced optical second harmonic generation in hybrid polymer nanoassemblies based on coupled surface plasmon resonance of a gold nanoparticle array

Appl. Phys. Lett. 89, 011903 (2006); 10.1063/1.2219086

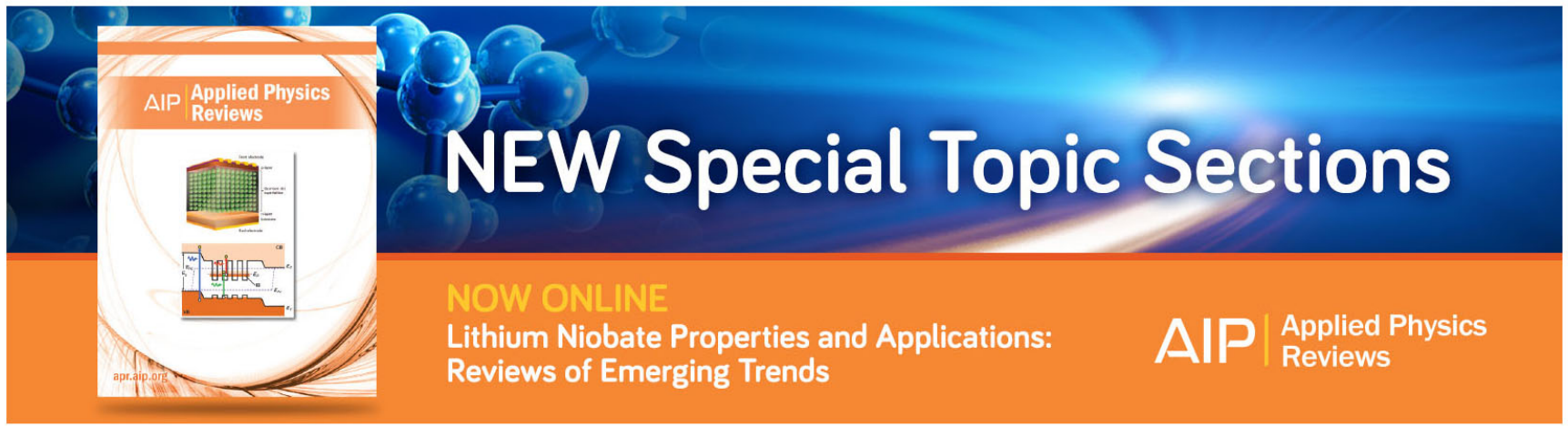




\title{
Resonant plasmonic nanoparticles for multicolor second harmonic imaging
}

\author{
Nicolò Accanto, ${ }^{1}$ Lukasz Piatkowski, ${ }^{1}$ Ion M. Hancu, ${ }^{1}$ Jan Renger, ${ }^{1}$ \\ and Niek F. van Hulst ${ }^{1,2, a)}$ \\ ${ }^{1}$ ICFO_Institut de Ciences Fotoniques, The Barcelona Institute of Science and Technology, \\ 08860 Castelldefels, Barcelona, Spain \\ ${ }^{2}$ ICREA—Institució Catalana de Recerca i Estudis Avançats, 08010 Barcelona, Spain
}

(Received 21 December 2015; accepted 16 February 2016; published online 26 February 2016)

\begin{abstract}
Nanoparticles capable of efficiently generating nonlinear optical signals, like second harmonic generation, are attracting a lot of attention as potential background-free and stable nano-probes for biological imaging. However, second harmonic nanoparticles of different species do not produce readily distinguishable optical signals, as the excitation laser mainly defines their second harmonic spectrum. This is in marked contrast to other fluorescent nano-probes like quantum dots that emit light at different colors depending on their sizes and materials. Here, we present the use of resonant plasmonic nanoparticles, combined with broadband phase-controlled laser pulses, as tunable sources of multicolor second harmonic generation. The resonant plasmonic nanoparticles strongly interact with the electromagnetic field of the incident light, enhancing the efficiency of nonlinear optical processes. Because the plasmon resonance in these structures is spectrally narrower than the laser bandwidth, the plasmonic nanoparticles imprint their fingerprints on the second harmonic spectrum. We show how nanoparticles of different sizes produce different colors in the second harmonic spectra even when excited with the same laser pulse. Using these resonant plasmonic nanoparticles as nano-probes is promising for multicolor second harmonic imaging while keeping all the advantages of nonlinear optical microscopy. (C) 2016 AIP Publishing LLC.

[http://dx.doi.org/10.1063/1.4942902]
\end{abstract}

Fluorescence microscopy is a widely used technique in biological imaging that enables the study of subcellular components and dynamical processes in vivo. A key challenge in fluorescence microscopy is to find suitable fluorescent markers or probes that when attached to specific proteins or compounds allow easy observation during a sufficient amount of time. ${ }^{1}$ The commonly used visible fluorescent markers are often problematic as they undergo photoinduced processes like blinking and bleaching that limit the stability of the experiment and the amount of detectable photons. Additionally, biological samples present autofluorescence in the visible range of the spectrum that may overlap with the emission of the markers, which is an important source of noise in the measurement. ${ }^{1-4}$ To overcome these limitations, researchers have recently devoted considerable attention to a different kind of marker: nanoparticles (NP) that can be excited via two-photon excitation and generate second harmonic ( $\mathrm{SH}$ ) radiation. ${ }^{2,3,5,6} \mathrm{NPs}$ like barium titanate $\left(\mathrm{BaTiO}_{3}\right)$, lithium niobate $\left(\mathrm{LiNbO}_{3}\right)$, iron iodate $\left(\mathrm{Fe}\left(\mathrm{IO}_{3}\right)_{3}\right)$, silicon carbide $(\mathrm{SiC})$, and zinc oxide $(\mathrm{ZnO})^{2,5}$ are stable over blinking and bleaching, which allows long time observations, emit in a relatively narrow spectral range, so they can be spectrally separated from sample autofluorescence, and can be excited with near infrared light, which limits photo-damage of the sample and increases the penetration depth for deep tissue imaging. ${ }^{7}$ However, the emission spectrum of these NPs is mainly determined by the excitation laser and does not change for different NPs. This is in contrast to other widely used fluorescent markers, like

\footnotetext{
${ }^{\text {a) }}$ Author to whom correspondence should be addressed. Electronic mail: Niek.vanHulst@ICFO.eu
}

quantum dots (QDs), which emit at different wavelengths according to their sizes and materials, even when excited with the same laser source. This allows for the labelling of different targets using different QDs and distinguish them with spectrally resolved detection, a technique that is often called multicolor fluorescence imaging. ${ }^{8,9}$ Multicolor imaging has so far been limited to fluorescent markers and not studied for SH NPs. In this work, we demonstrate that resonant plasmonic nanoparticles (RPNPs) can create distinct colors in the SH spectrum even when using the same excitation laser, which may allow multicolor SH imaging.

RPNPs are metallic NPs that efficiently interact with optical radiation taking advantage of localized surface plasmon resonances (LSPRs) in the visible and near infrared region of the spectrum. ${ }^{10-12}$ Among other appealing properties, RPNPs greatly enhance nonlinear optical effects. ${ }^{13,14}$ Second harmonic from RPNPs has been recently measured, ${ }^{15-18}$ and different methods to further enhance this nonlinear effect by engineering the RPNPs have been proposed. ${ }^{19-23}$ Interestingly, in a recent work ${ }^{24}$ we showed that the SH spectrum generated by RPNPs interacting with sufficiently broadband laser pulses depends on both the laser spectrum and the LSPR. This gives the unique possibility of distinguishing two different RPNPs, characterized by different LSPRs, based on their SH spectra. More importantly, by exploiting our ability of precisely phase shaping the ultrafast laser pulse, ${ }^{25}$ we describe here an experiment (sketched in Fig. 1) in which a suitable selection of the RPNPs combined with a tailored phase shape is used to generate different colors in the SH spectra of the RPNPs, which is at the basis of multicolor SH imaging. The biocompatibility of metallic $\mathrm{NPs}^{26}$ together with the versatility in the fabrication 


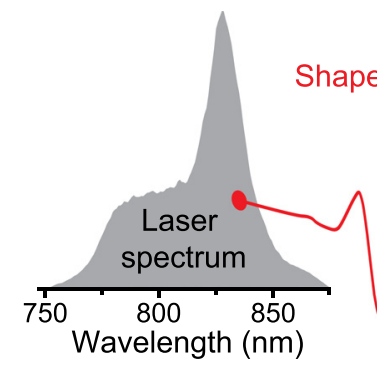

Shaped laser pulse

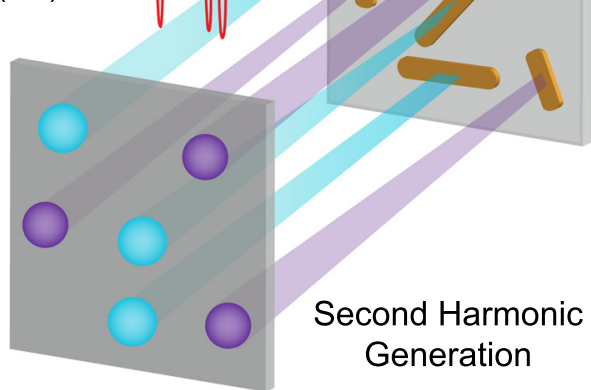

FIG. 1. Main concept of the experiment. A broadband laser pulse, after being shaped in its spectral phase by a pulse shaper, excites a sample of resonant plasmonic nanoparticles (RPNPs). If the RPNPs have different sizes, their plasmonic resonances are also spectrally shifted. In this case, for a suitable selection of the laser spectral phase, a different second harmonic spectrum is generated. Spectrally selective detection can be used to distinguish the RPNPs and create multicolor images.

processes makes the results presented here relevant for biological applications.

In the experiment, we used a $\sim 15 \mathrm{fs}$ titanium sapphire laser (Octavius 85M, Menlo Systems) tuned to a central wavelength of $\sim 830 \mathrm{~nm}$ with a bandwidth of $\sim 100 \mathrm{~nm}$ (see the spectrum in Fig. 1) as the broadband laser source. A pulse shaper arranged in a $4 \mathrm{f}$ configuration controls the spectral phase of the laser pulse, and a liquid crystal spatial light modulator (SLM) is used as the active element. The phase-shaped laser pulse is then sent to a two-photon microscope equipped with a high numerical aperture objective (1.3 NA) that focuses the laser beam on a sample containing the RPNPs. The sample can be raster scanned with respect to the objective using a nanometer precision piezoelectric scanner. The second harmonic generation (SHG) from the RPNPs is collected by the same objective in reflection geometry, spectrally filtered to reject the laser reflection and Rayleigh-scattered light, and sent to the photodetectors. Two-dimensional SH images of the sample are acquired using avalanche photodiodes (APDs), whereas a spectrometer equipped with an electron-multiplying charge-coupled device (emCCD) camera is used for spectral measurements.

We studied two different types of RPNPs: (i) silver nanorods of lengths ranging from 425 to $485 \mathrm{~nm}$ were used to demonstrate the experimental concept, i.e., the use of RPNPs with detuned LSPRs to produce different SH spectra; (ii) gold nano-rods of 100 to $130 \mathrm{~nm}$ in length allowed us to test the multicolor SH imaging technique on smaller NPs, therefore better suited for bio-labeling. Both types of RPNPs were prepared using electron beam lithography on a glass substrate coated with a $10 \mathrm{~nm}$ thin layer of indium-tin-oxide (ITO).

The silver RPNPs were arranged in arrays. Each array contains several nano-rods of identical lengths; changing array, the length of the RPNPs is changed to tune the spectral position of the LSPR across the laser spectrum. Here, we consider four different arrays of RPNPs (NP1, NP2, NP3, and NP4) of 425 to $485 \mathrm{~nm}$ in length. The RPNPs were fabricated to be resonant with different portions of the excitation laser: NP1 are resonant with the blue side of the laser spectrum, and the LSPRs continuously shift to the red for the other RPNPs. NP4 were fabricated to be resonant with the red side of the laser spectrum. The specific shape and material used for RPNPs were chosen to produce high SHG and present narrow LSPRs at the laser wavelengths. The silver RPNPs therefore were best suited to measure SH spectra of individual RPNPs on the CCD camera, which requires higher signal than in the case of imaging with an APD. The gold RPNP sample was instead prepared to have RPNPs with different lengths (and LSPRs) next to each other, to demonstrate the multicolor SH imaging technique.

First, we investigate the influence of the spectral position of the LSPRs on the SH spectra for the silver RPNPs. Typical scanning electron microscope (SEM) images of the fabricated RPNPs are shown in Fig. 2. The experiment is performed as follows: first, a Fourier-limited pulse (the shortest possible pulse for a given spectrum) is obtained at the sample position using the procedure of Ref. 25. The Fourier-limited pulse is then used to generate SH from the RPNPs. Detecting the SHG with the APD while scanning the sample position with respect to the objective allows us to acquire two dimensional $\mathrm{SH}$ images of the sample, as the one shown in Fig. 2(a) for one NP1 array. From such a scan, it is possible to select an individual RPNP and to direct its SHG to the spectrometer for spectral measurements. Because the SHG is a nonlinear process, its spectrum depends on the laser spectral phase, which gives us the possibility of acquiring different $\mathrm{SH}$ spectra from the same RPNP by changing the spectral phase applied with the SLM. In practice, for every RPNP, we collect 128 different $\mathrm{SH}$ spectra corresponding to 128 different applied phases. This phase scan is carried out following the
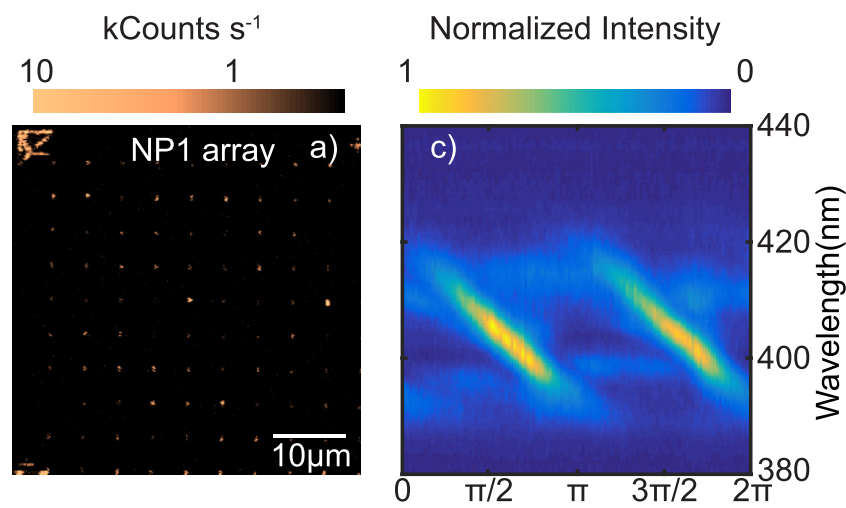

b)
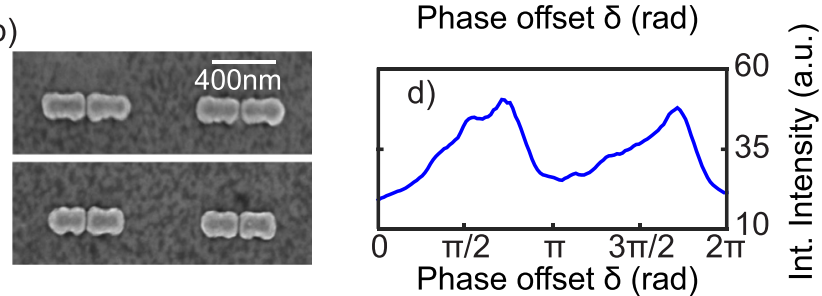

FIG. 2. (a) Two dimensional SH image of one NP1 array. Silver markers at the edges delimit the array. (b) Typical SEM images of the RPNPs. (c) Spectrally resolved MIIPS scan for a single NP1. (d) Spectrally integrated MIIPS scan resulting from the scan above. 
Multiphoton Intrapulse Interference Phase Scan (MIIPS) method, as described in Refs. 27-29.

During a MIIPS scan, a set of known sinusoidal spectral phases $\varphi(\omega, \delta)=\alpha \sin (\gamma \omega-\delta)$ is applied to the laser pulse by the SLM, as a function of the phase offset $\delta$ that varies from 0 to $2 \pi$, and the corresponding SH spectra are measured for every $\delta$. This produces spectrograms similar to the one reported in Fig. 2(c). Vertical cuts through the spectrogram correspond to SH spectra taken for different spectral phases applied by the SLM (different $\delta$ values). Fig. 2(d) shows the spectrally integrated MIIPS trace for the same RPNP, namely, the same scan as the one shown in Fig. 2(c), but integrated along the vertical axis. From this scan, one can better identify the positions of the absolute SH maxima and minima in the MIIPS trace.

We first discuss the possibility of achieving contrast in the total SH intensity using phase shaping. These results might provide a useful tool for distinguishing two different RPNPs when spectrally resolved detection is not available. It was shown in Ref. 2 that contrast in the SH intensity can be obtained between different materials $\left(\mathrm{BaTiO}_{3}\right.$ and $\left.\mathrm{SiC} \mathrm{NPs}\right)$ by changing the wavelength of a relatively narrowband laser from 800 to $880 \mathrm{~nm}$, as a consequence of different conversion efficiencies of the materials at the two wavelengths. However, this contrast mechanism requires two different lasers to be aligned in exactly the same way in an optical microscope, which is a cumbersome operation.

In Figs. 3(a)-3(c), we plot pairs of spectrally integrated MIIPS traces corresponding to four different RPNPs. Clearly, the positions of the SH maxima and minima change for different LSPRs, or in other words, the SH maxima and minima for different RPNPs are obtained for different values of $\delta$. Moreover, the relative change is bigger for LSPRs that are farther apart in the spectral domain. NP1 and NP4 were fabricated to be resonant on opposite sides of the laser spectrum,
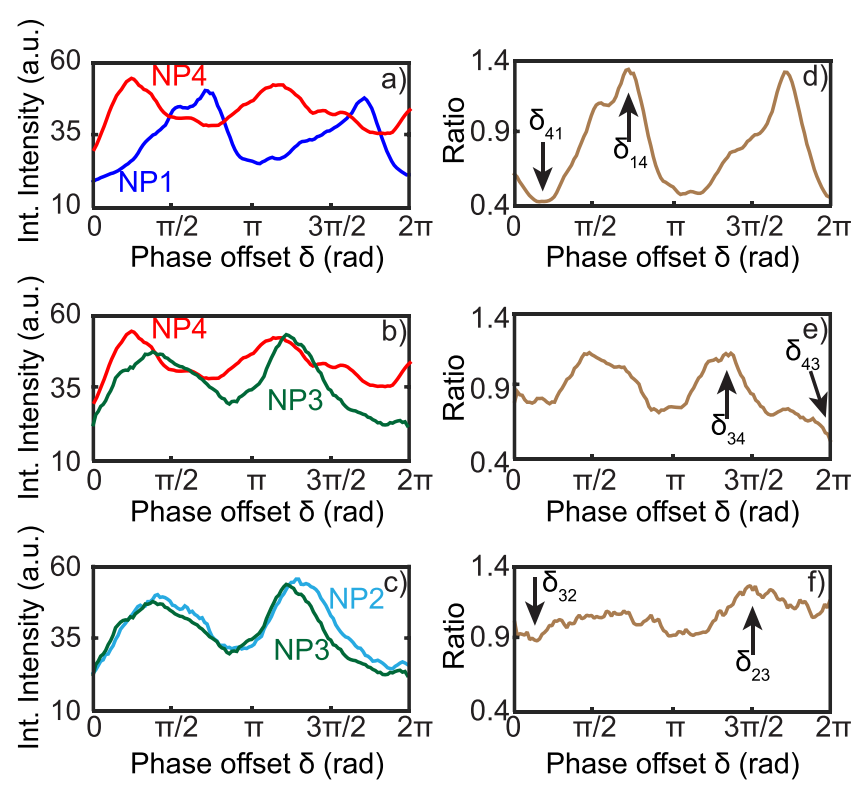

FIG. 3. (a), (b), and (c) Spectrally integrated MIIPS traces of the indicated antenna pairs. (d), (e), and (f) Ratio of the spectrally integrated SH intensity between NP1/NP4, NP3/NP4, and NP2/NP3 RPNPs, respectively, as a function of the phase offset. The arrows represent for each graph the points at which maximum and minimum ratio occur. The corresponding $\delta$ values generate maximum contrast between the two RPNPs. and the corresponding spectrally integrated MIIPS traces (Fig. 3(a)) are maximally shifted in the $\delta$ axis. The maxima and minima for these two RPNPs are completely out of phase. The relative shift is reduced for NP3 and NP4 and is almost zero in the case of NP2 and NP3, which are characterized by LSPRs that are much closer in the spectral domain. The fact that different RPNPs reach the SH maximum at different $\delta$ values gives the unique possibility of controlling the contrast in the SHG from different RPNPs simply by changing the laser spectral phase, which is less demanding than switching between two different laser wavelengths. This idea is supported by the graphs of Figs. 3(d)-3(f) where the ratio of the spectrally integrated MIIPS signal between NP1/NP4, NP3/NP4, and NP2/NP3 is plotted. One should interpret these graphs as follows: the ratio between the $\mathrm{SH}$ signals oscillates between $\sim 0.4$ and $\sim 1.3$ for NP1-NP4, between $\sim 0.6$ and $\sim 1.1$ for NP3-NP4, and is almost constant for NP2-NP3 as a function of $\delta$. As indicated in the graphs, we call $\delta_{14}$ the $\delta$ value at which the ratio NP1/NP4 is the largest (so that NP1 is brighter than NP4) and $\delta_{41}$ the $\delta$ value at which the ratio NP1/NP4 is the smallest (so that NP4 is brighter than NP1). The same rule applies for the other pairs; for instance, at the value $\delta_{34}, \mathrm{NP} 3$ is brighter than NP4 and at $\delta_{23}, \mathrm{NP} 2$ is brighter than NP3. Choosing the spectral phases corresponding to $\delta_{14}$ and $\delta_{41}$, or the analogous $\delta$ values for the other pairs, therefore generates the highest SH contrast in the RPNPs. The contrast increases for more spectrally separated LSPRs. Let NP1 $\left(\delta_{14}\right)$ be the total SH intensity from NP1 for the phase $\delta_{14}$, the contrast between NP1 and NP4 for the phases $\delta_{14}$ and $\delta_{41}$ is

$$
\frac{\mathrm{NP} 1\left(\delta_{14}\right)}{\mathrm{NP} 4\left(\delta_{14}\right)} \times \frac{\mathrm{NP} 4\left(\delta_{41}\right)}{\mathrm{NP} 1\left(\delta_{41}\right)}
$$

The contrast for other particles is defined in the same way. In the case of NP1-NP4, the maximum contrast reaches a factor of $\sim 3$; a factor of $\sim 2$ for NP3-NP4, and almost no contrast (or only very little) is obtainable for NP2-NP3.

These results demonstrate that RPNPs of different lengths coupled to ultrafast phase-controlled laser pulses can be used to obtain contrast in the SHG. However, this contrast is obtained upon the application of two different spectral phases on the SLM. For imaging purposes, this requires acquiring two different images of the sample, one for each spectral phase. Even if this might be simpler than changing the laser wavelength, it still requires two different actions. We now show that, once having two RPNPs that differ enough in their LSPRs, the best way of obtaining contrast in the SHG is to spectrally split the detection and separate the long and short wavelengths in the second harmonic (Fig. 4).

Given the laser spectrum shown in Fig. 1, we split the SHG between the blue $(\lambda<408 \mathrm{~nm})$ and the red $(\lambda>413 \mathrm{~nm})$ spectral window (as the dashed black line in Fig. 4), and for a specific pair of RPNPs, we look for the spectral phase (the $\delta$ value in the MIIPS scans) that maximizes the contrast between the two spectral windows. From Fig. 3, we concluded that the pair NP2-NP3 does not produce SH contrast; therefore, we focus on the pairs NP1-NP4 and NP3-NP4. As Fig. 4 clearly shows, for the pair NP1-NP4, one can find a $\delta$ value for which 


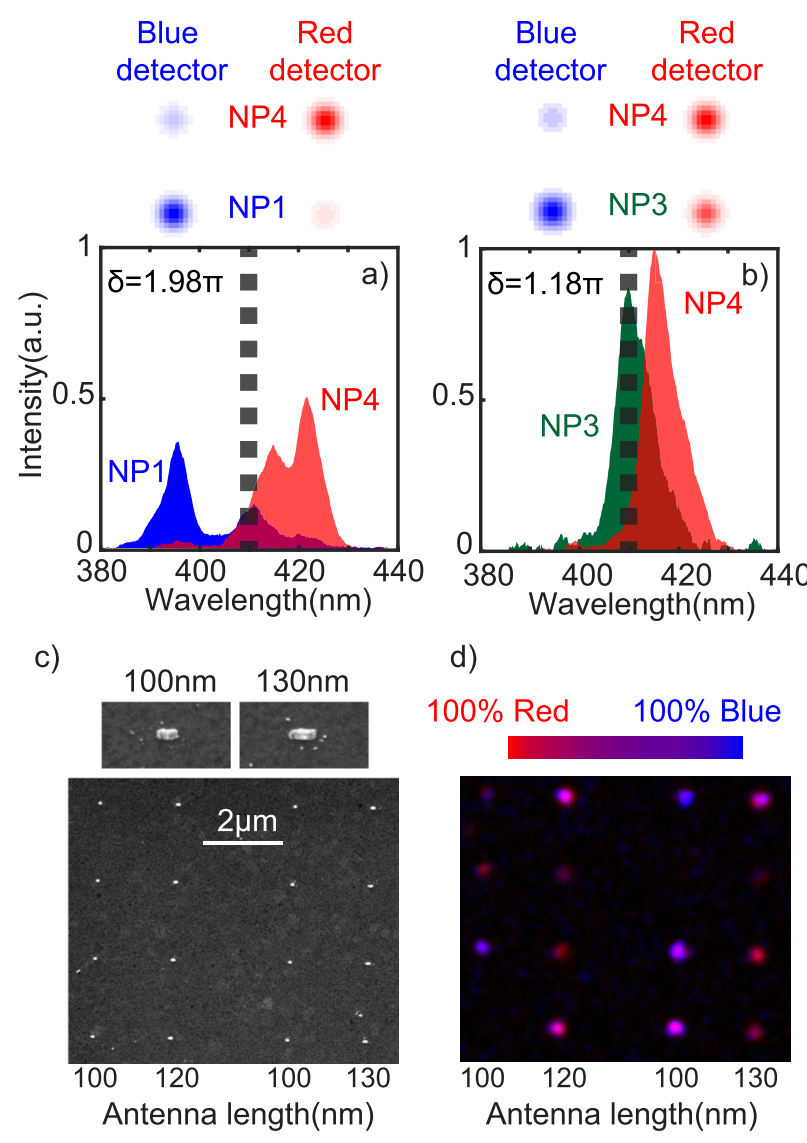

FIG. 4. Multicolor second harmonic imaging. (a) and (b) Second harmonic spectra for the indicated nano-rod pair that maximize the contrast between the blue $(\lambda<408 \mathrm{~nm})$ and red $(\lambda>413 \mathrm{~nm})$ side of the $\mathrm{SH}$ range. The black dashed line indicates the spectral position at which we assume to split the SH detection. The blue and red dots illustrate how the different RPNPs appear on spectrally selective two-dimensional images. (c) SEM images of an array of gold RPNPs of different lengths and higher resolution SEM images of the individual $100 \mathrm{~nm}$ and $130 \mathrm{~nm}$ RPNPs. (d) Two-color SH image of a nominally identical array acquired in one scan, showing contrast between different species, with longer RPNPs more red. The blue $(\lambda<400 \mathrm{~nm})$ and red $(400<\lambda<420 \mathrm{~nm})$ side of the SH-spectrum are pseudo-colored blue and red.

the SH spectra peak on the opposite sides of the SH range. If we take two detectors (two separate APDs) and send with a dichroic mirror the blue wavelength to the first APD ("blue" detector) and the red to the second APD ("red" detector), the spatial images obtained with the two APDs will look very different. At the top of Fig. 4(a), we draw how such experiment would look like. The red and blue spots represent the $\mathrm{SH}$ emitted by NP4 and NP1 and detected by the red and the blue detectors, respectively. The spatially integrated intensity of the spots corresponds to the spectrally integrated SH spectra of Fig. 4(a) in the corresponding spectral window. Clearly, the blue detector is mainly sensitive to the SH from NP1 whereas the red detector mainly sees NP4. With NP1blue and NP1red, the total SH intensity from NP1 in the blue and red spectral windows, respectively, the contrast between NP1 and NP4 in the two different detectors is defined as

\section{$\frac{\text { NP1blue }}{\text { NP4blue }} \times \frac{\text { NP4red }}{\text { NP1red }}$}

For the NP1-NP4 pair, from Fig. 4(a), we calculate a total spectrally resolved contrast of $\sim 50$. As already shown in Fig. 3, for the other RPNP pairs, the contrast decreases as the spectral position of the corresponding LSPRs gets closer to each other. For NP2-NP4 (Fig. 4(b)), we can find a $\delta$ value for which the contrast between the blue and the red detectors is $\sim 6$, and for NP2-NP3, almost no spectral contrast can be achieved.

Finally, we have applied the multicolor SH imaging concept to the gold NP sample, prepared to have NPs of different lengths close to each other. The arrangement of the sample and the shape of the NPs are clearly resolved in the SEM images (Fig. 4(c)). In the same array columns of $100 \mathrm{~nm}$ gold NPs alternate with columns of $120 \mathrm{~nm}$ and $130 \mathrm{~nm}$ NPs. The $100 \mathrm{~nm}$ NPs are resonant with the blue side of the laser spectrum, and the resonance shifts to the red for longer particles. The NPs were imaged with two APDs, detecting the blue $(\lambda<400 \mathrm{~nm})$ and the red (400 $<\lambda<420 \mathrm{~nm}$ ) parts of the SH spectrum, respectively. The two independent $\mathrm{SH}$ images were represented with a blue and a red pseudo-color scale, respectively, and overlaid to construct the two-color image of Fig. 4(d). A clear contrast between the $100 \mathrm{~nm}$ and the longer RPNPs is revealed. The $100 \mathrm{~nm}$ NPs appear blue to violet in the image, while the 120 and $130 \mathrm{~nm}$ NPs appear purple to red. It is also apparent that some NPs do not completely follow this trend or appear dimmer. This difference is attributed to variations in the nanofabrication of the RPNPs. The amorphous gold nanorods contain single crystal particles, and a size difference of only $10 \mathrm{~nm}$ is sufficient to shift the spectrum. Evidently different species of RPNPs can be readily distinguished by spectrally resolved $\mathrm{SH}$ detection. Moreover, the presented SH imaging is of direct relevance as it demonstrates that small, and therefore better suited for labeling purposes, NPs can be used for multicolor SH imaging.

From the results reported in Fig. 4, we can conclude that by using suitable RPNPs together with the appropriate laser spectral phase, we can generate different colors in the $\mathrm{SH}$ spectra, which allows multicolor SH imaging using metallic nano-particles. This has two great advantages with respect to the approach illustrated in Fig. 3 or to the contrast mechanism of Ref. 2: (i) it generates much larger contrast, which allows different RPNPs to be better resolved, and (ii) when applied to microscopy, it only requires a single acquisition and two detectors with different spectral filters, as the different colors in the $\mathrm{SH}$ are generated for the same excitation conditions. We also wish to stress here that this kind of spectral contrast can be only obtained by using broadband laser pulses, with a bandwidth larger than the LSPRs, while the use of narrowband pulses of different wavelengths would not generate spectral contrast between different RPNPs. In order to push this contrast further, even broader laser spectra, or narrower LSPRs would be beneficial.

In summary, we have shown that resonant plasmonic nano-particles greatly affect nonlinear optical signals such as second harmonic generation. Using broadband phase controlled laser pulses, we exploit this property to generate distinguishable SH spectra from different nano-particles. Owing to the versatility of the fabrication processes, and the understanding of plasmonic effects developed in recent years, a great variety of SH responses in nanometric volumes may be designed. The biocompatibility of metallic nanoparticles makes the results presented here relevant for 
biological imaging purposes. Exploiting this concept, we have demonstrated multicolor second harmonic imaging using RPNPs. These plasmonic probes allow maintaining all the advantages of nonlinear microscopy, while adding spectral selectivity in the second harmonic generation.

The authors would like to thank Pablo M. de Roque for useful discussions and help in the interpretation of the data and Vikas Remesh for kind help with the imaging experiment. L.P. acknowledges financial support from the Marie-Curie International Fellowship COFUND and the ICFOnest program. This research was funded by the European Commission (ERC Adv. Grant 247330-NanoAntennas and ERC Adv. Grant 670949-LightNet), Fundació CELLEX (Barcelona) and Plan Nacional Project FIS2012-35527, co-funded by FEDER.

${ }^{1}$ C. Vonesch, F. Aguet, J. L. Vonesch, and M. Unser, IEEE Signal Process. Mag. 23, 20 (2006).

${ }^{2}$ P. Pantazis, J. Maloney, D. Wu, and S. E. Fraser, Proc. Natl. Acad. Sci. U. S. A. 107, 14535 (2010).

${ }^{3}$ B. E. Cohen, Nature 467, 407 (2010).

${ }^{4}$ N. Billinton and A. W. Knight, Anal. Biochem. 291, 175 (2001).

${ }^{5}$ D. Staedler, T. Magouroux, R. Hadji, C. Joulaud, J. Extermann, S. Schwung, S. Passemard, C. Kasparian, G. Clarke, M. Gerrmann, R. L. Dantec, Y. Mugnier, D. Rytz, D. Ciepielewski, C. Galez, S. GerberLemaire, L. Juillerat-Jeanneret, L. Bonacina, and J.-P. Wolf, ACS Nano 6, 2542 (2012).

${ }^{6}$ F. Wang, R. Deng, J. Wang, Q. Wang, Y. Han, H. Zhu, X. Chen, and X. Liu, Nat. Mater. 10, 968 (2011).

${ }^{7}$ F. Helmchen and W. Denk, Nat. Methods 2, 932 (2005).

${ }^{8}$ W. C. W. Chan, D. J. Maxwell, X. Gao, R. E. Bailey, M. Han, and S. Nie, Curr. Opin. Biotechnol. 13, 40 (2002).

${ }^{9}$ B. A. Kairdolf, A. M. Smith, T. H. Stokes, M. D. Wang, A. N. Young, and S. Nie, Annu. Rev. Anal. Chem. 6, 143 (2013).
${ }^{10}$ L. Novotny and N. van Hulst, Nat. Photonics 5, 83 (2011).

${ }^{11}$ J.-J. Greffet, Science 308, 1561 (2005).

${ }^{12}$ P. Biagioni, J.-S. Huang, and B. Hecht, Rep. Prog. Phys. 75, 024402 (2012).

${ }^{13}$ M. Castro-Lopez, D. Brinks, R. Sapienza, and N. F. van Hulst, Nano Lett. 11, 4674 (2011).

${ }^{14}$ M. Kauranen and A. V. Zayats, Nat. Photonics 6, 737 (2012).

${ }^{15}$ J. Butet, J. Duboisset, G. Bachelier, I. Russier-Antoine, E. Benichou, C. Jonin, and P.-F. Brevet, Nano Lett. 10, 1717 (2010).

${ }^{16}$ A. Slablab, L. Le Xuan, M. Zielinski, Y. de Wilde, V. Jacques, D. Chauvat, and J. F. Roch, Opt. Express 20, 220 (2012).

${ }^{17}$ H.-D. Deng, G.-C. Li, Q.-F. Dai, M. Ouyang, S. Lan, V. A. Trofimov, and T. M. Lysak, Nanotechnology 24, 075201 (2013).

${ }^{18}$ M. D. McMahon, D. Ferrara, C. T. Bowie, R. Lopez, and R. F. Haglund, Jr., Appl. Phys. B 87, 259 (2007).

${ }^{19}$ H. Aouani, M. Navarro-Cia, M. Rahmani, T. P. H. Sidiropoulos, M. Hong, R. F. Oulton, and S. A. Maier, Nano Lett. 12, 4997 (2012).

${ }^{20}$ B. Metzger, L. Gui, J. Fuchs, D. Floess, M. Hentschel, and H. Giessen, Nano Lett. 15, 3917 (2015).

${ }^{21}$ K. Thyagarajan, J. Butet, and O. J. F. Martin, Nano Lett. 13, 1847 (2013).

${ }^{22}$ M. Celebrano, X. Wu, M. Baselli, S. Großmann, P. Biagioni, A. Locatelli, C. De Angelis, G. Cerullo, R. Osellame, B. Hecht, L. Duò, F. Ciccacci, and M. Finazzi, Nat. Nanotechnol. 10, 412 (2015).

${ }^{23}$ R. Czaplicki, H. Husu, R. Siikanen, J. Mäkitalo, M. Kauranen, J. Laukkanen, J. Lehtolahti, and M. Kuittinen, Phys. Rev. Lett. 110, 093902 (2013).

${ }^{24}$ N. Accanto, L. Piatkowski, J. Renger, and N. F. van Hulst, Nano Lett. 14, 4078 (2014).

${ }^{25}$ N. Accanto, J. B. Nieder, L. Piatkowski, M. Castro-Lopez, F. Pastorelli, D. Brinks, and N. F. van Hulst, Light Sci. Appl. 3, e143 (2014).

${ }^{26}$ E. Hutter and D. Maysinger, Microsc. Res. Tech. 74, 592 (2011).

${ }^{27}$ Y. Coello, V. V. Lozovoy, T. C. Gunaratne, B. Xu, I. Borukhovich, C.H. Tseng, T. Weinacht, and M. Dantus, J. Opt. Soc. Am B 25, A140 (2008).

${ }^{28}$ V. V. Lozovoy, I. Pastirk, and M. Dantus, Opt. Lett. 29, 775 (2004).

${ }^{29}$ B. Xu, J. M. Gunn, J. M. Dela Cruz, V. V. Lozovoy, and M. Dantus, J. Opt. Soc. Am. B 23, 750 (2006). 